\title{
Canonical and non-canonical JAK/STAT transcriptional targets may be involved in distinct and overlapping cellular processes
}

\author{
Amy Tsurumi ${ }^{1,2,3^{*}}$ (D), Connie Zhao ${ }^{4}$ and Willis X. Li ${ }^{5}$
}

\begin{abstract}
Background: The Janus kinase-signal transducer and activator of transcription (JAK/STAT) pathway has been wellcharacterized as a crucial signal transduction cascade that regulates vital biological responses including development, immunity and oncogenesis. Additionally to its canonical pathway that uses the phosphorylated form of the STAT transcription factor, recently the non-canonical pathway involving heterochromatin formation by unphosphorylated STAT was recently uncovered. Considering the significant role of the JAK/STAT pathway, we used the simple Drosophila system in which the non-canonical pathway was initially characterized, to compare putative canonical versus non-canonical transcriptional targets across the genome. We analyzed microarray expression patterns of wildtype, Jak gain- and loss-of-function mutants, as well as the Stat loss-of-function mutant during embryogenesis, since the contribution of the canonical signal transduction pathway has been well-characterized in these contexts. Previous studies have also demonstrated that Jak gain-of-function and Stat mutants counter heterochromatin silencing to de-repress target genes by the non-canonical pathway.

Results: Compared to canonical target genomic loci, non-canonical targets were significantly more associated with sites enriched with heterochromatin-related factors $(p=0.004)$. Furthermore, putative canonical and non-canonical transcriptional targets identified displayed some differences in biological pathways they regulate, as determined by Gene Ontology (GO) enrichment analyses. Canonical targets were enriched mainly with genes relevant to development and immunity, as expected, whereas the non-canonical target gene set mainly showed enrichment of genes for various metabolic responses and stress response, highlighting the possibility that some differences may exist between the two loci.

Conclusions: Canonical and non-canonical JAK/STAT genes may regulate distinct and overlapping sets of genes and may perform specific overall functions in physiology. Further studies at different developmental stages, or using distinct tissues may identify additional targets and provide insight into which gene targets are unique to the canonical or non-canonical pathway.
\end{abstract}

Keywords: JAK, STAT, Non-canonical JAK/STAT signaling, Drosophila, Development, Signal transduction, Epigenetics, Heterochromatin

\footnotetext{
* Correspondence: tsurumi@research.mgh.harvard.edu

'Department of Surgery, Massachusetts General Hospital, 50 Blossom St.,

Thier 340, Boston, MA 02114, USA

${ }^{2}$ Department of Microbiology and Immunology, Harvard Medical School, 77

Ave. Louis Pasteur, Boston, MA 02115, USA

Full list of author information is available at the end of the article
} 


\section{Background}

The Janus kinase-signal transducer and activator of transcription (JAK/STAT) signaling pathway was originally characterized in mammals as an intracellular signaling pathway regulating cytokine signaling [1-5] and was later found to be highly conserved in various organisms, including Drosophila [6-9]. Four JAKs and seven STAT gene products have been identified in mammals, whereas in Drosophila, the pathway components are comprised of simply one JAK and one STAT, making it an excellent model system to study their biological roles $[8,10]$. In both mammals and Drosophila, the canonical signaling is initiated by the binding of an extracellular ligand to transmembrane receptors that induce dimerization and activate the JAKs associated with these receptors. Then, the activated JAKs phosphorylate tyrosine residues on the cytoplasmic tails of the receptors that serve as docking sites for cytoplasmic STAT transcription factors, which then are phosphorylated, dimerize and translocate to the nucleus to activate transcription of target genes [11-13]. It has been wellestablished across various species that the canonical JAK/STAT pathway performs essential roles in development, immune signaling and cancers by its direct transcriptional activation of target genes [8, 14-18].

The non-canonical JAK/STAT pathway was initially identified in Drosophila, in which both the STAT transcription factor and the protein inhibitor of activated STAT homologue (dPIAS) were found to be suppressors of variegation, a heterochromatin-mediated phenomenon [19-21]. Heterochromatin-mediated gene silencing mechanisms involving the main non-histone key protein, Heterochromatin Protein 1a (HP1a), otherwise known as Suppressor of varigation 205 (Su(var)205) in Drosophila, is highly conserved across species $[19,22,23]$. HP1a recognizes and is recruited to specific genomic loci by diand tri-methylated histone 3 lysine 9 (H3K9), which is regulated by the $\mathrm{Su}(\mathrm{var}) 3-9$ methyltransferase [22, 24, 25]. The biological role of such non-canonical JAK/STAT pathway was first meticulously characterized in a tumor model of Drosophila, in which unphosphorylated STAT was found to exist in the nucleus and to have the ability to stabilize heterochromatin and suppress tumor growth [12, $19,26]$. Moreover, it was found that heterochromatin formation and stabilization by unphosphorylated STAT protects genome stability [27] and prolongs lifespan [28]. The pivotal role of unphosphorylated STAT in heterochromatin maintenance and tumor suppression was also recapitulated in mammals [29]. Other groups have reported that unphosphorylated STAT proteins can translocate to and prominently exist in the nucleus in various mammalian cells at quiescence, when STAT proteins are not phosphorylated [30-35]. Several studies have demonstrated functions of STAT1, STAT3, STAT5 and STAT6 in mammals that involve mechanisms that are distinct to their well-established canonical transcriptional pathway [36-45]. Additional studies have characterized the involvement of the linker histone $\mathrm{H} 1$ in non-canonical JAK/ STAT mechanisms, further suggesting the central role of unphosphorylated STAT in epigenetic regulation [46]. In Drosophila, a connection between JAK/STAT signaling and chromatin remodeling has also been suggested by the finding that the transcriptional repressor Ken that recruits a nucleosome remodeling factor (NURF), shares binding sequences to suppress STAT-mediated transcription of innate immune genes [47]. The epigenetic control of heterochromatin formation and remodeling are crucial for key nuclear processes including gene silencing, chromosomal packaging and segregation during mitosis, genome stability and cell differentiation [27, 48, 49]. Additionally to its biological role in oncogenesis, heterochromatin maintenance in Drosophila was found to mediate lifespan [28] and stem cell maintenance [50], as well as implicated in mammalian aging [51]. These observations further underline the crucial role of the non-canonical JAK/STAT pathway in various vital biological processes, additionally to its formerly established canonical signal transduction mechanisms.

Considering the well-established role of the canonical JAK/STAT signaling cascade and the recent characterization of non-canonical JAK/STAT that is distinct and appear to perform specific roles in physiology and pathogenesis, it is important to identify target genes and genomic loci that may be regulated by one or the other. While there are previous studies that have undertaken a systematic approach to uncover JAK/STAT pathway regulators [52, 53], there has not been a study addressing differences and similarities between canonical and non-canonical targets. We therefore took a genomewide approach to identify target loci pertaining to each, using Drosophila as a simple model system, in which both the canonical and non-canonical JAK/STAT have been particularly well-characterized. We analyzed genome-wide transcriptional targets of the Jak gain- and loss-of-function mutants, and the Stat loss-of-function mutant during embryogenesis, as previous studies have addressed the role of the canonical signal transduction in these mutants and furthermore, it has also been found that Jak gain-of-function and Stat loss-of-function mutants counter heterochromatin silencing to de-repress target genes by the non-canonical pathway $[19,26]$. We found non-canonical targets to correlate with increased overlaps with the distribution of HP1a, Su(var)3-9 and H3K9me3, which are hallmarks of heterochromatin formation. Moreover, our study demonstrates that while canonical targets tend to be enriched with genes relevant to development and immune response, non-canonical target genes tend to be more enriched with GO terms 
related to metabolism and stress response, thus possibly revealing a previously unidentified putative link between the non-canonical JAK/STAT pathway and metabolism and stress response, mediated by heterochromatin. We therefore conclude that a significant number of distinct canonical and non-canonical targets appear to exist, additionally to overlapping processes and loci. Further experimental results comparing transcriptome alterations in conjunction with chromatin immunoprecipitation from the same embryonic samples will be beneficial for confirming the conclusions from these analyses.

\section{Results}

\section{Analysis of various JAK/STAT mutant embryo} transcriptome changes relative to wildtype show clustering based on genotype and embryo stage

We collected early Drosophila embryos (0-12 h eggs) from the following four different genotypes: Jak gain- (hop ${ }^{\text {Tum-ll }}$ $\left.{ }^{+}\right)$, and loss-of-function $\left(\mathrm{hop}^{3 /+}\right)$, Stat loss-of-function heterozygotes $\left(\right.$ Stat $\left.92 E^{06346 /+}\right)$, and the Stat loss-of-function

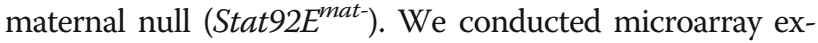
periments to compare the transcriptome of these embryos (Additional file 1: Table S1). Gene expression levels were calculated as fold changes relative to wildtype $\left(w^{1118}\right)$ embryos of the same stage. Hierarchical clustering analyses indicated that hop ${ }^{\text {Tum-l/+}}$ and Stat $92 E^{\text {mat- }}$ embryos were most dissimilar, with many genes expressed in the opposite manner (Fig. 1A), consistent with gain- and loss-offunction of the JAK/STAT pathway. Analysis of differentially regulated probe sets found clustering of hop ${ }^{T u m-l /+}$ and $h o p^{3 /+}$ together, then Stat92 $E^{06346 /+}$, and Stat92E $E^{\text {mat }}$ (Fig. 1A; Additional file 2: Table S2). Next, we assessed the number of overlapping probe sets between $S t a t 92 E^{\text {mat }}$ and Stat $92 E^{06346 /+}$ upregulated and downregulated sets of genes (Additional file 3: Table S3). For the upregulated probe sets there were 49 that were shared between the 381 Stat $92 E$ mat- and 579 Stat $92 E^{06346 /+}$ and for the downregulated set, there were 47 that were shared between the 788 Stat92Emat- and 640 Stat $92 E^{06346 /+}$ significantly downregulated probe sets (Fig. 1B). Since Stat92 $E^{06346 /+}$ heterozygous embryos are expected to undergo normal development in spite of reduced Stat gene activity, the difference between the transcriptomes of Stat $92 E^{\text {mat- }}$ and Stat $92 E^{06346 /+}$ might be due to a failure in activation of the zygotic genome in Stat92 $E^{\text {mat- }}$ embryos, as we have previously shown [54]. The differentially regulated genes include many of the known canonical JAK/STAT pathway targets genes, such as the Turandot (Tot) family of humoral factors [55] (Additional file 2: Table S2). We validated the differential expression of several of these known JAK/STAT target genes using RTPCR in the hop $p^{T u m-l /+}$ overexpression mutants compared to wildtype (Additional file 4: Fig. S1). These results suggest that the activation status and levels of JAK and STAT affect the transcriptome of the early Drosophila embryo. In our
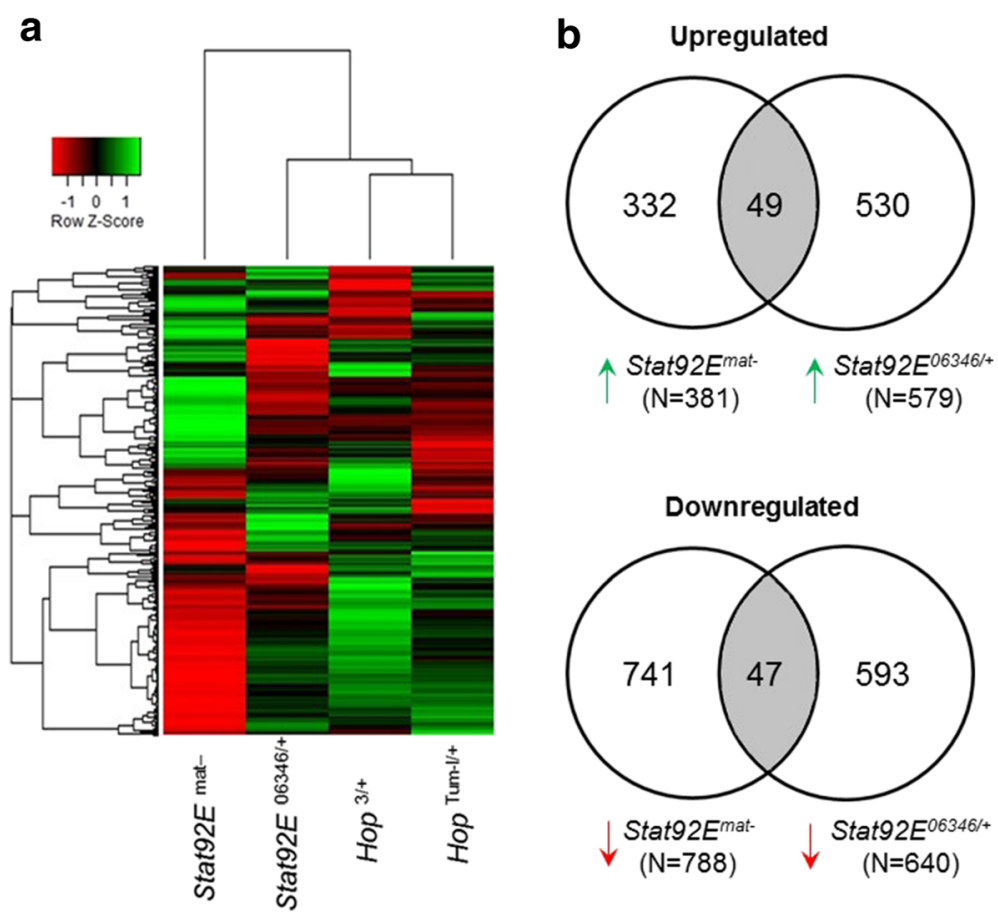

Fig. 1 Hierarchical clustering of Jak gain-of-function and Stat loss-of-function mutants show separation by gene and stage of the embryo. (a) Differentially regulated genes were clustered by Pearson's correlation complete distance separation. (b) Overlaps between Stat92 $E^{\text {mat- }}$ and Stat92 $E^{06346 /+}$ embryos were determined and suggest that a large number of genes are regulated stage-specifically 
previously published publication, we have validated reduced expression in the early loss-of-function embryo of the $d p p$, $\mathrm{Kr}, \mathrm{tll}$, and eve target genes that we have also found in our current microarray analysis [54].

\section{Canonical versus non-canonical JAK/STAT targets can be inferred from differential transcriptional analyses across the genome of various mutants}

A hallmark of the noncanonical STAT pathway is that loss of STAT and JAK overactivation both result in reduced heterochromatin levels and previously demonstrated to be relevant in multiple heterochromatininvolved processes [26-28]. Thus, genes normally repressed by heterochromatin would be depressed as a result of reduced heterochromatin. These studies showed that in contrast, in the canonical pathway, loss of STAT and JAK overactivation have opposite effects on STAT target gene expression.

Since the hierarchical clustering analysis showed large differences in overall transcriptomes of Stat $92 E^{\text {mat- }}$ and Stat $92 E^{06346 /+}$ embryos, we aimed to differentiate between canonical versus non-canonical JAK/STAT pathway by analyzing significantly regulated probe sets of Jak gain- $\left(h o p^{\text {Tum-l/+ }}\right)$ and loss-of-function $\left(h o p^{3 /+}\right)$ and Stat loss-of-function (Stat92 $E^{06346 /+}$ ) separately from the maternal null $\left(\right.$ Stat $\left.2 E^{\text {mat }}\right)$. For the putative canonical targets assessing overlaps among hop ${ }^{\text {Tum-l/+ }}$ upregulated, $h o p^{3 /+}$ downregulated and Stat $92 E^{06346 /+}$ downregulated genes, we found 221 putative probe sets and for the putative non-canonical targets assessing overlaps among hop ${ }^{\text {Tum- } /+l}$ upregulated, hop ${ }^{3 /+}$ downregulated and Stat $92 E^{06346 /+}$ upregulated genes, we found 371 putative probe sets (Fig. 2A). While comparing putative canonical targets by assessing overlaps among hop ${ }^{\text {Tum-l/+ }}$ upregulated, hop ${ }^{3 /+}$ downregulated and Stat $92 E^{\text {mat- }}$ downregulated genes, we found 66 putative probe sets and for the putative non-canonical targets assessing overlaps among hop ${ }^{\text {Tum-l/+}}$ upregulated, hop ${ }^{3 /+}$ downregulated and Stat $92 E^{\text {mat- }}$ upregulated genes, we found another $66 \mathrm{pu}-$ tative probe sets (Fig. 2B). The total number of unique probe sets for further analyses was 258 putative canonical and 409 putative non-canonical probe sets (Additional file 5: Table S4).

\section{Non-canonical targets are significantly more enriched with heterochromatin markers}

Since the distinct mode of the non-canonical JAK/STAT pathway involves heterochromatin stabilization by unphosphorylated STAT by its interaction with HP1a, we hypothesized that we would observe increased association of putative non-canonical target loci with key heterochromatin markers, HP1a, Su(var)3-9 and H3K9me3 [19, 26, 27, 29]. We therefore identified overlaps with the publicly available modENCODE ChIP-seq database annotating loci enriched with HP1a, Su(var)39 and H3K9me3 in various wildtype background samples at different developmental stages and Drosophila cell culture [56]. Among probe sets that were classified as
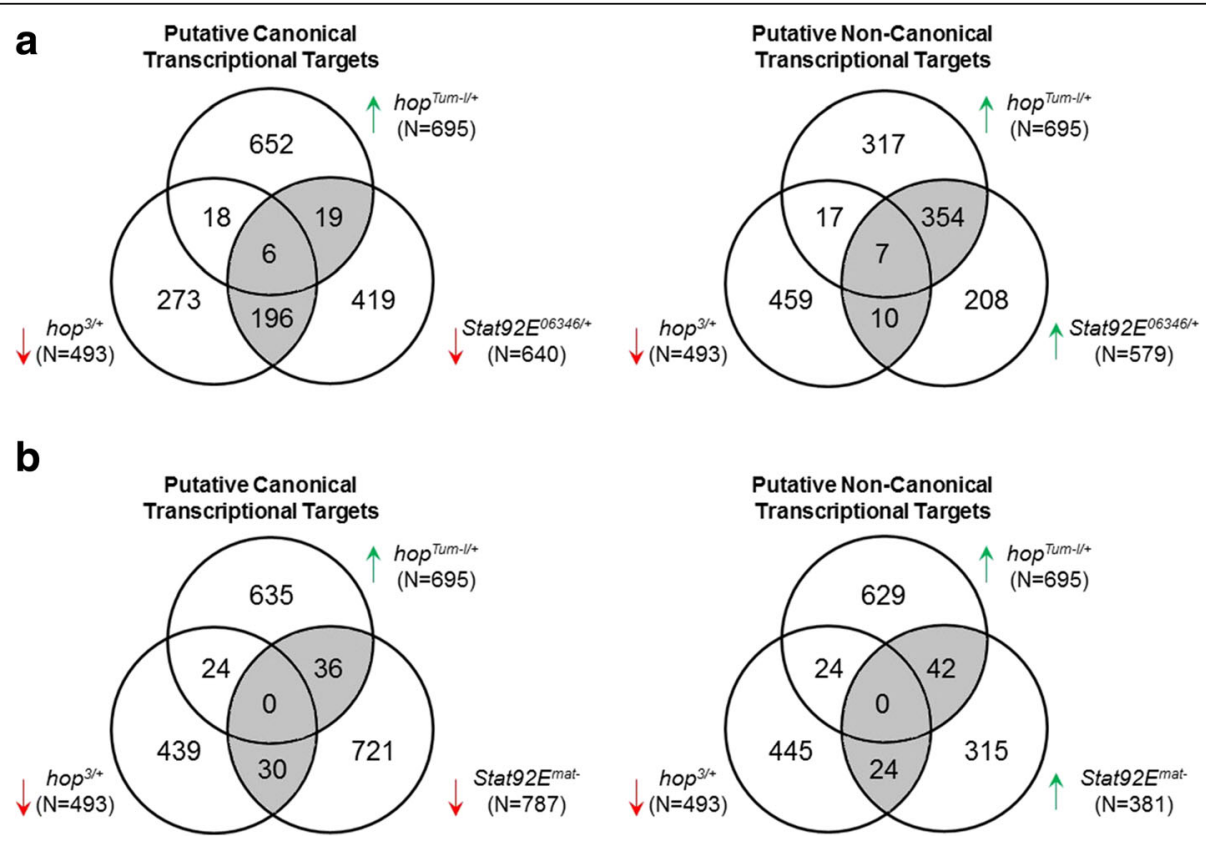

Fig. 2 Biologically relevant overlapping probe sets of putative canonical and non-canonical transcriptional targets inferred by microarray. Significant genes were assessed for overlaps among the Jak-Stat mutants previously determined to be relevant for the canonical versus non-canonical heterochromatin-mediated silencing mechanism $[19,26]$ for (a) embryo samples and (b) maternal null and embryo samples 
putative canonical targets by our transcriptional analyses, 25.6\% had HP1a, Su(var)3-9 and/or H3K9me3 enriched site overlap and $1.6 \%$ mapped to transposable elements, compared to $35.9 \%$ and $2.2 \%$ respectively in noncanonical targets. Our results comparing the putative canonical versus the putative non-canonical targets we identified from our transcriptome analyses thus indicate that as expected, there was significant difference between these two groups, in which the putative non-canonical transcriptional target group had a larger number of overlap sites with HP1a, Su(var)3-9 and/or H3K9me3, or are transposable element sites, compared to the group classified as canonical transcriptional targets (38.1\% versus $27.1 \%, p=0.004$ Fisher's exact test) (Fig. 3).

\section{Canonical and non-canonical targets have distinct bio- logical roles}

After having classified probe sets to canonical versus non-canonical JAK/STAT pathway transcriptional target genes, we sought the biological significance of these different targets. We used the DAVID Gene Ontology database to determine biological functions and pathways that are enriched for the canonical and non-canonical target gene panel $[57,58]$. Canonical targets appeared to be mainly involved in development and innate immunity, as expected for their well-established role, as seen by the top 20 fold enrichment of GO Biological Functions terms (Fig. 4). Ten out of 20 top enriched terms were relevant to development, including "larval chitin-based cuticle development," "establishment of epithelial cell apical/basal polarity," "body morphogenesis," "chitinbased cuticle development," "metamorphosis," "negative regulation of cell proliferation," "chorion-containing eggshell formation," "wing disc development," "imaginal disc-derived wing morphogenesis" and "multicellular organism reproduction." Terms relevant to innate immune response were also found four times, including "innate immune response," "response to bacterium," "Toll signaling pathway" and "defense response to Gram-positive bacterium." Other enrichment terms suggested a role for the canonical pathway in olfactory and sensory response, including "detection of chemical stimulus involved in sensory perception of taste," and "sensory perception of smell." Additional GO terms suggested transport mechanisms, including, "microtubule-based process" and "transmembrane transport" and others included "cellular response to heat" and "proteolysis."

On the other hand, non-canonical targets were enriched mainly with terms relevant to metabolism, including "glucose metabolic process," "ecdysteroid metabolic process," "pyruvate metabolic process," and stress response including "detection of temperature stimulus involved in thermoception," "cold acclimation," "thermotaxis," "mechanosensory behavior," and "cellular response to oxidative stress." Female sex and egg development also appeared to be prevalent among the $\mathrm{GO}$ enriched terms, including "imaginal disc-derived female genitalia development," "eggshell chorion assembly," "vitellogenesis" and "vitelline membrane formation involved in chorion-containing eggshell formation." Regulation of visual perception also appeared multiple times including "compound eye retinal cell programmed cell death," "visual perception" and "deactivation of rhodopsin mediated signaling." "Regulation of G-protein coupled receptor protein signaling pathway" was also among the top fold enrichment. The observation that the highest GO term fold enrichment was seen for "negative regulation of RNA splicing" is noteworthy, and may be related to the role of the non-canonical pathway in epigenetic signaling. GO terms related to chorion formation were found in both canonical and non-canonical targets, suggesting that while a large number of target

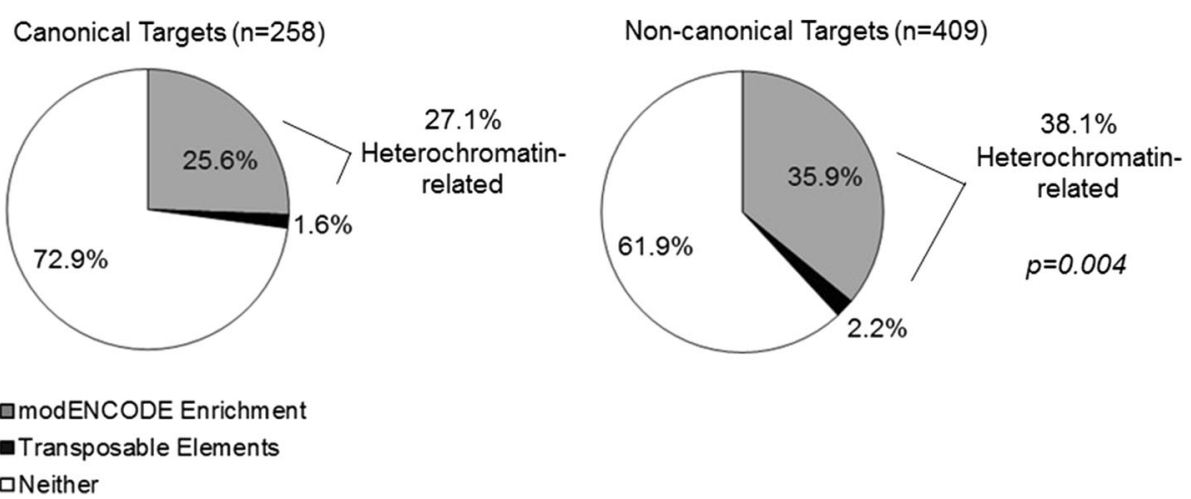

Fig. 3 Non-canonical target sites have significantly more overlaps with heterochromatin sites. For both embryonic and maternal transcripts, overlaps between genomic loci corresponding to the probe set annotation and HP1a, Su(var)3-9 and/or H3K9me3 enriched loci listed in the relevant modENCODE database were tabulated. Probe sets annotated as transposable elements were also considered as heterochromatin sites. Non-canonical target probe sets had significantly higher proportion of such heterochromatin-related sites compared to canonical targets $(p=0.004$, Fisher's exact two-tailed test) 


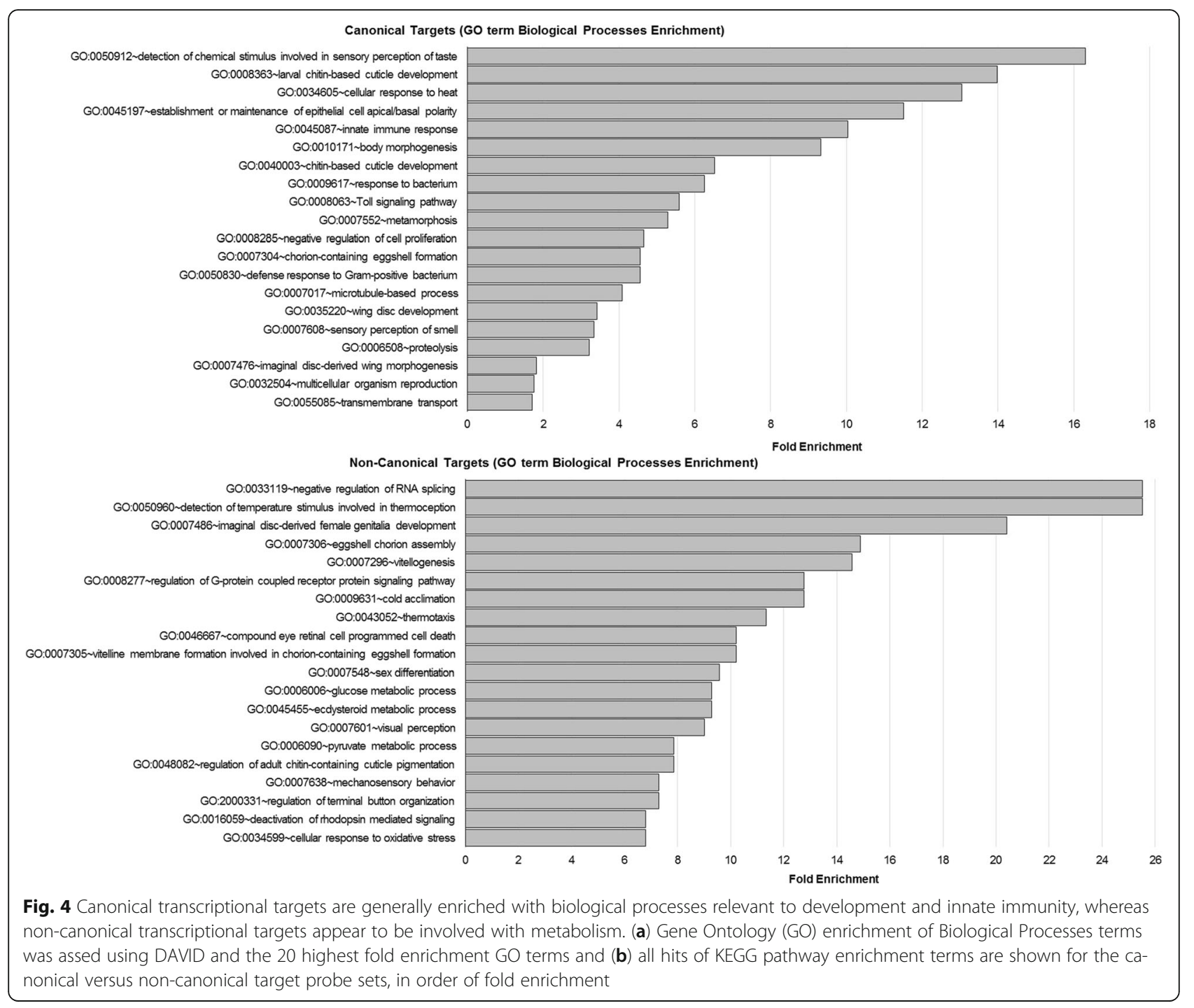

loci distinct to canonical or non-canonical pathways exist, there is also a possibility of shared processes and targets.

\section{Discussion}

Our current study aimed to differentiate between canonical versus non-canonical JAK/STAT target genes in Drosophila by using a genome-wide transcriptional analysis approach. The use of the Drosophila system with a simple JAK/STAT system that involves only one JAK and one STAT with mutant lines readily available facilitated this current study that aimed to distinguish between the two pathways by transcriptional analysis. The genetic analysis of the non-canonical JAK/STAT pathway and the establishment of the paradigm of unphosphorylated STAT as a key player in epigenetic regulation in the nucleus has been conducted in Drosophila $[19,26]$. We therefore performed genome-wide transcriptome analyses to characterize transcriptional targets of relevant Jak and Stat mutants in this study. Furthermore, we quantified overlaps with ChIP-seq peaks of the major heterochromatin factors of HP1a, $\mathrm{Su}$ (var)3-9 and H3K9me3 binding sites using available modENCODE data. Our findings that putative noncanonical transcriptional targets had significantly more heterochromatin marker overlaps compared to the putative canonical targets given this study design supports the notion that while canonical targets rely mainly on classic signaling transduction modes to activate transcriptional target genes via phosphorylated and dimerized STAT, the non-canonical epigenetic mechanism via unphosphorylated STAT operates in a distinct manner, by its interaction with HP1a and heterochromatin associated factors. Additional studies conducting chromatin immunoprecipitation experiments in conjunction with transcriptomic analyses using samples of the exact same developmental stage would confirm the conclusions drawn from this study. 
In flies and other organisms, HPla-mediated heterochromatin maintenance has been implicated as a regulator of the aging process $[28,51]$. A specific underlying association between heterochromatin regulation, metabolism, aging and oncogenesis can be inferred from multiple studies in different organisms. In S. cerevisiae, metabolism, genomic instability and cell life span through replication have been linked to heterochromatin [59]. Furthermore, it was found from a later study in C.elegans, that mutants of the HP1a homologue, HPL-2 altered germline gene expression that controls the switch to the dauer state, as well as longevity and lipid metabolism [60]. These observations are consistent with the GO term enrichment of the non-canonical JAK/ STAT pathway we observed in the current study, in which we found among the highest fold enrichment, gene groups related to various metabolic pathways, stress response, the female genitalia and egg development. It has been shown previously that unphosphorylated STAT can be protective against genome instability and oncogenesis by its interaction with HP1a, and may further suggest that the enrichment in various metabolism-related genes we have observed especially in the non-canonical heterochromatin-mediated pathway may be related to the aforementioned links among these various processes described in different species [27, 29]. Stress response terms were also frequently found to be among the top 20 fold enrichment, mostly regarding cold temperature sensing and response, as well as response to oxidative stress. It has been shown previously in Arabidopsis that epigenetic regulation of heterochromatin markers occur at repetitive elements in response to cold-stress, as well as activation of transcription of heterochromatic loci induced by stress [61, 62]. Furthermore, a link between oxidative stress and heterochromatin stabilization has been described and shown to provide genome protection [63]. It is also interesting to note that the highest fold enrichment GO term found among non-canonical targets was "negative regulation of RNA splicing." Considering the epigenetic role of the non-canonical pathway, it is conceivable that additionally to heterochromatin maintenance, the non-canonical pathway may coordinate RNA splicing. In contrast to the putative non-canonical targets where GO terms related to metabolism, stress response and female sexrelated terms were among the most enriched terms, GO term enrichment of the canonical target gene group were related to the well-established roles of the JAK/ STAT pathway, development and innate immune response, as expected. Transcriptional regulation of genes related to development and innate immunity by the JAK/STAT pathway is known to be highly conserved across species [12, 64]. Indeed, we found $T o t C$ and $T o t X$ in our analyses of putative transcriptional target genes inferred from the microarray and modEncode overlaps (Additional file 5: Table S4). These observations provide further support for the idea that our approach may be effective in uncovering additional target genes, both canonical and non-canonical.

In this study, we utilized relatively young mutant embryos laid between 0 and $12 \mathrm{~h}$. On the other hand, the modENCODE dataset that we inferred heterochromatinrelated factors varied, from early embryo to the late larval stage of different genetic backgrounds, as well as various cell lines. Our conclusions would therefore be strengthened by additional experimental studies using specific genetic backgrounds, same tissues and temporally regulated samples for further transcriptional analyses, coupled by chromatin immunoprecipitation of relevant heterochromatin factors of the corresponding samples. Such future studies would enable us to identify additional targets, and perform an increasingly meticulous analysis to differentiate between the canonical versus non-canonical JAK/STAT pathway. Nevertheless, our current study supports the notion that canonical versus non-canonical JAK/STAT pathway may regulate numerous biologically distinct processes additionally to some possible overlaps and it would be beneficial to conduct further studies that establish methods that distinguish between the two pathways.

\section{Conclusions}

We conducted a genome-wide analysis comparing putative canonical versus non-canonical JAK/STAT pathway transcriptional targets during early Drosophila development. Our findings of differences between canonical versus non-canonical JAK/STAT pathway targets and specific loci regulated across the genome give us insights into the significant and distinct biological roles of each that may exist, additionally to possible overlapping targets.

\section{Methods}

Fly stocks/genetics and RNA sample preparation

All crosses were carried out at $25{ }^{\circ} \mathrm{C}$ on standard cornmeal/agar medium. Fly stocks of $w^{1118}$, hop $3 / F M 7 c$, Stat92E $E^{06346} / T_{M} 3$, FRT $^{82 B}$ [ovo $\left.^{D 1}, w^{+}\right] / T M 3$, and hop ${ }^{\text {Tum- }}$ ${ }^{l} / F M 7 c$ were obtained from the Bloomington Drosophila Stock Center (Bloomington, IN). For the preparation of heterozygous embryos, females from the mutant stocks were crossed with $w^{1118}$ males and the resulting hop ${ }^{3 /+}$, Stat $92 E^{06346 /+}$ or hop ${ }^{\text {Tum-l/+ }}$ progeny were used to produce embryos, which were collected between 0 and $12 \mathrm{~h}$ after egg laying on apple agar plates with yeast paste. To generate Stat $92 E^{\text {mat- }}$ embryos, hsp70-flp; FRT ${ }^{82 B}$ Stat $92 E^{06346} / T M 3$ females were crossed to hsp70Flp; FRT ${ }^{82 B}\left[\mathrm{ovo}^{D 1}, \mathrm{w}^{+}\right] / T M 3$ males. Third instar larval progenies were heat-shocked at $37^{\circ} \mathrm{C}$ for $2 \mathrm{~h}$ daily over 
3 to 4 days, and resulting adult females of the genotype hsp70-flp; FRT ${ }^{82 B}$ Stat $92 E^{06346} / F R T^{82 B}\left[{ }^{2} v o^{D 1}, w^{+}\right]$which were used to produce embryos lacking in maternal Stat $92 E$ gene products, as described previously in the dominant female-sterile "germline clone" technique [65]. Stat92 $E^{\text {mat- }}$ and control $w^{1118}$ were collected between 1 and $2 \mathrm{~h}$ after egg laying on apple agar plates with yeast paste. The embryos were washed twice with deionized water and total RNA was prepared using the RNeasy Plus Mini kit (Qiagen) according to the manufacturer's manual. RNA quality was assessed using the Agilent 2100 Bioanalyzer and the RNA 6000 Nano kit (Agilent Technologies Inc., Palo Alto, CA).

\section{Microarray analyses}

To prepare microarray samples from the RNA prepared, $200 \mathrm{ng}$ of total RNA was used to prepare biotin-labeled RNA using Ambion MessageAmp Premier RNA Amplification Kit (Applied Biosystems, Forster City, CA). Briefly, the first strand of cDNA was synthesized using ArrayScript reverse transcriptase and an oligo(dT) primer bearing a T7 promoter. Then DNA polymerase I was used (in the presence of E. coli RNase $\mathrm{H}$ and DNA ligase) to convert single-stranded cDNA into doublestranded DNA (dsDNA), which was then used as a template for in vitro transcription in a reaction containing biotin-labeled UTP and T7 RNA Polymerase to generate biotin-labeled antisense RNA (aRNA). Twenty $\mu \mathrm{g}$ of labeled aRNA was fragmented and $15 \mu \mathrm{g}$ of the fragmented aRNA was hybridized to Affymetrix Drosophila Genome 2.0 Array Chips according to the manufacterer's Manual (Affymetrix, Santa Clara, CA). Array Chips were stained with streptavidin-phycoerythrin, followed by an antibody solution (anti-streptavidin) and a second streptavidin-phycoerythrin solution, performed by a GeneChip Fluidics Station 450. The Array Chips were scanned with the Affymetrix GeneChip Scanner 3000. For the numerical conversion to expression intensity and Present/Absent calls employing MAS5 [66] (Additional file 1: Table S1), the Genespring software (Agilent Technologies Inc., Palo Alto, CA) or the $\mathrm{R}$ package Affy was used [67, 68]. R version 3.1.3 was used for the analyses.

For each mutant genotype, control probe sets were filtered, as well as those where the wild-type and respective mutant intensities all had the "Absent" call. The top 10th percentile upregulated and downregulated $\log _{2}$ fold change of all probes were found to be 1.027 and -1.047 , respectively and therefore the 2 -fold change cut-off was considered to be significantly differentially regulated genes for each mutant genotype (Additional file 2: Table S2). Pearson's correlation with complete distance separation was used for the clustering and heatmap representation of the differentially regulated probe sets. For conducting RT-PCR, 0-12 h embryos were collected and total RNA was harvarested using the RNeasy kit (Qiagen). The SuperScript ${ }^{\text {tu }}$ III Reverse Transcriptase kit (Invitrogen) was used to generate cDNA as a template for semi-quantitative PCR.

HP1a and $\mathrm{Su}(\mathrm{var}) 3-9$ binding and the heterochromatic H3K9me3 enriched sites were obtained from the publicly available modENCODE ChIP-seq database for comparison with genomic sites associated with the relevant probe sets identified from the microarray analysis [56]. The following modENCODE data files were used: for HP1a binding sites, \#3956 (Oregon ${ }^{\mathrm{R}}$ 14-16 h embryo), \#323 (S2 cells), \#955 (Oregon ${ }^{\mathrm{R}}$ 3rd instar larvae), \#2074 (S2 cells), \#2665 (Oregon ${ }^{\mathrm{R}} 2-4 \mathrm{~h}$ embryo), \#2666 (BG3c2 cells), \#2668 (S2 cells) and \#3956 (Oregon ${ }^{\mathrm{R}} 14-16 \mathrm{~h}$ embryo), for $\mathrm{Su}$ (var)3-9 binding sites, \#952 (BG3-c2) and \#2673 (S2), and for H3K9me3 enrichment, \#971 (yellow cinnabar brown speck 0-4 h embryo) and \#4939 (Oregon ${ }^{\mathrm{R}} 14-16 \mathrm{~h}$ embryo). In our study, if one or more enrichment sites were found to overlap with the annotated genomic locus indicated by the microarray data, the gene was deemed to be relevant to heterochromatin. For the overlap between probe sets upregulated by hopTum-l/+ and downregulated by hop $p^{3 /+}$, probe sets overlapping with downregulated Stat $92 E^{06346 /+}$ was categorized as putative canonical targets, whereas those upregulated were categorized as non-canonical targets. Similarly, Sta$t 92 E^{\text {mat- }}$ was also analyzed by taking into consideration, overlaps with hop Tum-l/+ upregulated and hop ${ }^{3 /+}$ downregulated probe sets. Probe sets where the Stat $92 E^{06346 /+}$ and Stat $92 E^{\text {mat- }}$ showed opposite trends were removed from the analyses, as well as $h o p^{T u m-l /+}$ and $h o p^{3 /+}$ overlaps without significant Stat $92 E^{06346 /+}$ or Stat $92 E^{\text {mat- }}$ differential expression.

The two-tailed Fisher's exact test was used to determine significance between the difference in the number of probe sets for which their relevant sites overlapped with HP1a, Su(var)3-9 and/or H3K9me3 enriched sites and transposable elements comparing putative canonical versus non-canonical targets. Database for Annotation, Visualization and Integrated Discovery (DAVID), version 6.8 Beta was used for functional annotation and assessing the top 20 Fold Enrichment of Gene Ontology terms of putative canonical and non-canonical sets [57].

\section{Additional files}

\footnotetext{
Additional file 1: Normalized dataset. (XLSX $3589 \mathrm{~kb})$

Additional file 2: Significant up- and down-regulated fold change probe sets of each mutant genotype. (XLSX $308 \mathrm{~kb}$ )
}

Additional file 3: Overlaps of significantly changed mutant probe sets and putative canonical/non-canonical transcriptional target classification. (XLSX $138 \mathrm{~kb})$ 
Additional file 4: Fig. S1. Validation of transcriptional upregulation of known target genes in hop ${ }^{\text {Tum/t+ }}$ embryo samples. RT-PCR was conducted on 0-12 h W $W^{1118}$ wildtype control, or hop ${ }^{\text {Tum/ }}$ embryo collection to assess the upregulation of previously known JAK-STAT target genes. (JPEG $27 \mathrm{~kb}$ )

Additional file 5: Heterochromatin marker modENCODE enrichment and transcriptional target overlaps. (XLSX $56 \mathrm{~kb}$ )

\section{Abbreviations}

ChIP-seq: Chromatin immunoprecipitation-sequencing; H3K9me3: Histone 3 Lysine 9 tri-methylation; HP1a: Heterochromatin Protein 1a; JAK/STAT: Janus kinase-signal transducer and activator of transcription; Su(var)205: Suppressor of varigation 205

\section{Acknowledgements}

We thank the Bloomington Drosophila Stock Center for antibodies and Drosophila strains. AT was supported by the Shriners Hospitals Research Fellowship \#84293. We would like to acknowledge the input and editing efforts of Yashoda Dhole, Laura Goodfield and Paris Karniadakis.

\section{Funding}

This work was supported by an NIH grant (R01CA131326) to WXL.

\section{Availability of data and materials}

The datasets used in this study are included within the Additional files.

\section{Authors' contributions}

AT prepared the samples. CZ processed the microarray. AT and WXL analyzed and interpreted the data. AT and WXL prepared the manuscript draft. All authors reviewed the manuscript. All authors read and approved the final manuscript.

\section{Ethics approval and consent to participate}

Not applicable.

\section{Consent for publication}

Not applicable.

\section{Competing interests}

The authors declare that they have no competing interests.

\section{Publisher's Note}

Springer Nature remains neutral with regard to jurisdictional claims in published maps and institutional affiliations.

\section{Author details}

'Department of Surgery, Massachusetts General Hospital, 50 Blossom St., Thier 340, Boston, MA 02114, USA. ${ }^{2}$ Department of Microbiology and Immunology, Harvard Medical School, 77 Ave. Louis Pasteur, Boston, MA 02115, USA. 'Shriners Hospitals for Children-Boston ${ }^{\circledR}, 51$ Blossom St, Boston, MA 02114, USA. ${ }^{4}$ Genomics Resource Center, The Rockefeller University, New York, NY, USA. ${ }^{5}$ Department of Medicine, University of California at San Diego, 9500 Gilman Dr, La Jolla, CA 92093, USA.

\section{Received: 11 November 2016 Accepted: 14 August 2017}

Published online: 11 September 2017

\section{References}

1. Darnell JE Jr, Kerr IM, Stark GR. Jak-STAT pathways and transcriptional activation in response to IFNs and other extracellular signaling proteins. Science. 1994;264(5164):1415-21.

2. Levy DE, Darnell JE Jr. Stats: transcriptional control and biological impact. Nat Rev Mol Cell Biol. 2002;3(9):651-62.

3. Bromberg J, Darnell JE Jr. The role of STATs in transcriptional control and their impact on cellular function. Oncogene. 2000;19(21):2468-73.

4. Aaronson DS, Horvath CM. A road map for those who don't know JAKSTAT. Science. 2002;296(5573):1653-5.

5. Schindler C, Shuai K, Prezioso VR, Darnell JE JJ. Interferon-dependent tyrosine phosphorylation of a latent cytoplasmic transcription factor. Science. 1992;257(5071):809-13.
6. Arbouzova NI, Zeidler MP. JAK/STAT signalling in drosophila: insights into conserved regulatory and cellular functions. Development. 2006;133(14):2605-16.

7. Hou XS, Melnick MB, Perrimon N. Marelle acts downstream of the drosophila HOP/JAK kinase and encodes a protein similar to the mammalian STATs. Cell. 1996;84(3):411-9.

8. Yan R, Small S, Desplan C, Dearolf CR, Darnell JE Jr. Identification of a Stat gene that functions in drosophila development. Cell. 1996;84(3):421-30.

9. Harrison DA, McCoon PE, Binari R, Gilman M, Perrimon N. Drosophila unpaired encodes a secreted protein that activates the JAK signaling pathway. Genes Dev. 1998;12(20):3252-63.

10. Binari R, Perrimon N. Stripe-specific regulation of pair-rule genes by hopscotch, a putative Jak family tyrosine kinase in drosophila. Genes Dev. 1994:8(3):300-12.

11. Brown S, Zeidler MP. Unphosphorylated STATs go nuclear. Curr Opin Genet Dev. 2008;18(5):455-60.

12. Li WX. Canonical and non-canonical JAK-STAT signaling. Trends Cell Biol. 2008:18(11):545-51.

13. Silver-Morse L, Li WX. JAK-STAT in heterochromatin and genome stability. JAKSTAT. 2013:2(3):e26090.

14. Bromberg J. Stat proteins and oncogenesis. J Clin Invest. 2002;109(9):1139-42.

15. Dutta P, Sabri N, Li J, Li WX. Role of STAT3 in lung cancer. JAKSTAT. 2014; 3(4):e999503.

16. Yu H, Jove R. The STATs of cancer-new molecular targets come of age. Nat Rev Cancer. 2004;4(2):97-105.

17. Itle JN. The Stat family in cytokine signaling. Curr Opin Cell Biol. 2001;13(2):211-7.

18. Shuai K, Liu B. Regulation of JAK-STAT signalling in the immune system. Nat Rev Immunol. 2003;3(11):900-11.

19. Shi S, Calhoun HC, Xia F, Li J, Le L, Li WX. JAK signaling globally counteracts heterochromatic gene silencing. Nat Genet. 2006;38(9):1071-6.

20. Hari KL, Cook KR, Karpen GH. The drosophila Su(var)2-10 locus regulates chromosome structure and function and encodes a member of the PIAS protein family. Genes Dev. 2001;15(11):1334-48.

21. Betz A, Lampen N, Martinek S, Young MW, Darnell JE Jr. A drosophila PIAS homologue negatively regulates stat92E. Proc Natl Acad Sci U S A. 2001; 98(17):9563-8

22. Black JC, Van Rechem C, Whetstine JR. Histone lysine methylation dynamics: establishment, regulation, and biological impact. Mol Cell. 2012;48(4):491-507.

23. Bartova E, Krejci J, Harnicarova A, Galiova G, Kozubek S. Histone modifications and nuclear architecture: a review. J Histochem Cytochem. 2008:56(8):711-21.

24. Jenuwein T, Allis CD. Translating the histone code. Science. 2001;293(5532):1074-80.

25. Dutnall RN. Cracking the histone code: one, two, three methyls, you're out! Mol Cell. 2003;12(1):3-4.

26. Shi S, Larson K, Guo D, Lim SJ, Dutta P, Yan SJ, Li WX. Drosophila STAT is required for directly maintaining HP1 localization and heterochromatin stability. Nat Cell Biol. 2008;10(4):489-96.

27. Yan SJ, Lim SJ, Shi S, Dutta P, Li WX. Unphosphorylated STAT and heterochromatin protect genome stability. FASEB J. 2011;25(1):232-41.

28. Larson K, Yan SJ, Tsurumi A, Liu J, Zhou J, Gaur K, Guo D, Eickbush TH, Li WX. Heterochromatin formation promotes longevity and represses ribosomal RNA synthesis. PLoS Genet. 2012:8(1):e1002473.

29. Hu X, Dutta P, Tsurumi A, Li J, Wang J, Land H, Li WX. Unphosphorylated STAT5A stabilizes heterochromatin and suppresses tumor growth. Proc Natl Acad Sci U S A. 2013;1 10(25):10213-8.

30. Chatterjee-Kishore M, Wright KL, Ting JP, Stark GR. How Stat1 mediates constitutive gene expression: a complex of unphosphorylated Stat1 and IRF1 supports transcription of the LMP2 gene. EMBO J. 2000;19(15): $4111-22$.

31. Iyer J, Reich NC. Constitutive nuclear import of latent and activated STAT5a by its coiled coil domain. FASEB J. 2008;22(2):391-400.

32. Liu L, McBride KM, Reich NC. STAT3 nuclear import is independent of tyrosine phosphorylation and mediated by importin-alpha3. Proc Natl Acad Sci U S A. 2005;102(23):8150-5.

33. Vinkemeier U. Getting the message across, STAT! Design principles of a molecular signaling circuit. J Cell Biol. 2004;167(2):197-201.

34. Yang J, Liao X, Agarwal MK, Barnes L, Auron PE, Stark GR. Unphosphorylated STAT3 accumulates in response to IL- 6 and activates transcription by binding to NFkappaB. Genes Dev. 2007:21(11):1396-408.

35. Yue H, Li W, Desnoyer R, Karnik SS. Role of nuclear unphosphorylated STAT3 in angiotensin II type 1 receptor-induced cardiac hypertrophy. Cardiovasc Res. 2010;85(1):90-9. 
36. Gough DJ, Corlett A, Schlessinger K, Wegrzyn J, Larner AC, Levy DE. Mitochondrial STAT3 supports Ras-dependent oncogenic transformation. Science. 2009;324(5935):1713-6.

37. Khan R, Lee JE, Yang YM, Liang FX, Sehgal PB. Live-cell imaging of the association of STAT6-GFP with mitochondria. PLOS One. 2013;8(1):e55426.

38. Kumar A, Commane M, Flickinger TW, Horvath CM, Stark GR. Defective TNFalpha-induced apoptosis in STAT1-null cells due to low constitutive levels of caspases. Science. 1997;278(5343):1630-2.

39. Lee JE, Yang YM, Liang FX, Gough DJ, Levy DE, Sehgal PB. Nongenomic STAT5-dependent effects on Golgi apparatus and endoplasmic reticulum structure and function. Am J Physiol Cell Physiol. 2012;302(5): C804-20.

40. Ng DC, Lin BH, Lim CP, Huang G, Zhang T, Poli V, Cao X. Stat3 regulates microtubules by antagonizing the depolymerization activity of stathmin. J Cell Biol. 2006;172(2):245-57.

41. Nilsson J, Bjursell G, Kannius-Janson M. Nuclear Jak2 and transcription factor NF1-C2: a novel mechanism of prolactin signaling in mammary epithelial cells. Mol Cell Biol. 2006;26(15):5663-74.

42. Silver DL, Naora H, Liu J, Cheng W, Montell DJ. Activated signal transducer and activator of transcription (STAT) 3: localization in focal adhesions and function in ovarian cancer cell motility. Cancer Res. 2004;64(10):3550-8.

43. Verma NK, Dourlat J, Davies AM, Long A, Liu WQ, Garbay C, Kelleher D, Volkov Y. STAT3-stathmin interactions control microtubule dynamics in migrating T-cells. J Biol Chem. 2009;284(18):12349-62.

44. Wang YZ, Wharton W, Garcia R, Kraker A, Jove R, Pledger WJ. Activation of Stat3 preassembled with platelet-derived growth factor beta receptors requires Src kinase activity. Oncogene. 2000;19(17):2075-85.

45. Wegrzyn J, Potla R, Chwae YJ, Sepuri NB, Zhang Q, Koeck T, Derecka M, Szczepanek K, Szelag M, Gornicka A, et al. Function of mitochondrial Stat3 in cellular respiration. Science. 2009:323(5915):793-7.

46. Xu N, Emelyanov AV, Fyodorov DV, Skoultchi Al. Drosophila linker histone $\mathrm{H} 1$ coordinates STAT-dependent organization of heterochromatin and suppresses tumorigenesis caused by hyperactive JAK-STAT signaling. Epigenetics Chromatin. 2014;7:16.

47. Kwon SY, Xiao H, Glover BP, Tjian R, Wu C, Badenhorst P. The nucleosome remodeling factor (NURF) regulates genes involved in drosophila innate immunity. Dev Biol. 2008;316(2):538-47.

48. Grewal SI, Jia S. Heterochromatin revisited. Nat Rev Genet. 2007:8(1):35-46

49. Grewal SI, Moazed D. Heterochromatin and epigenetic control of gene expression. Science. 2003:301(5634):798-802.

50. Xing Y, Li WX. Heterochromatin components in germline stem cell maintenance. Sci Rep. 2015;5:17463.

51. Tsurumi A, Li WX. Global heterochromatin loss: a unifying theory of aging? Epigenetics. 2012;7(7):680-8.

52. Baeg GH, Zhou R, Perrimon N. Genome-wide RNAi analysis of JAK/STAT signaling components in drosophila. Genes Dev. 2005;19(16):1861-70.

53. Muller P, Kuttenkeuler D, Gesellchen V, Zeidler MP, Boutros M. Identification of JAKSSTAT signalling components by genome-wide RNA interference. Nature. 2005;436(7052):871-5.

54. Tsurumi A, Xia F, Li J, Larson K, LaFrance R, Li WX. STAT is an essential activator of the zygotic genome in the early drosophila embryo. PLoS Genet. 2011;7(5):e1002086.

55. Bina S, Wright VM, Fisher KH, Milo M, Zeidler MP. Transcriptional targets of drosophila JAK/STAT pathway signalling as effectors of haematopoietic tumour formation. EMBO Rep. 2010;11(3):201-7.

56. Mod EC, Roy S, Ernst J, Kharchenko PV, Kheradpour P, Negre N, Eaton ML, Landolin JM, Bristow CA, Ma L, et al. Identification of functional elements and regulatory circuits by drosophila modENCODE. Science. 2010;330(6012):1787-97.

57. Dennis G, Jr., Sherman BT, Hosack DA, Yang J, Gao W, Lane HC, Lempick RA: DAVID: database for annotation, visualization, and integrated discovery. Genome Biol 2003, 4(5):P3.

58. Huang da W, Sherman BT, Lempicki RA. Systematic and integrative analysis of large gene lists using DAVID bioinformatics resources. Nat Protoc. 2009:4(1):44-57.

59. Bitterman KJ, Medvedik O, Sinclair DA. Longevity regulation in Saccharomyces Cerevisiae: linking metabolism, genome stability, and heterochromatin. Microbiol Mol Biol Rev. 2003;67(3):376-99. table of contents

60. Meister P, Schott S, Bedet C, Xiao Y, Rohner S, Bodennec S, Hudry B, Molin $L$, Solari F, Gasser SM, et al. Caenorhabditis Elegans heterochromatin protein 1 (HPL-2) links developmental plasticity, longevity and lipid metabolism. Genome Biol. 2011;12(12):R123.
61. Hu Y, Zhang L, He S, Huang M, Tan J, Zhao L, Yan S, Li H, Zhou K, Liang Y, et al. Cold stress selectively unsilences tandem repeats in heterochromatin associated with accumulation of H3K9ac. Plant Cell Environ. 2012;35(12):2130-42.

62. Tittel-Elmer M, Bucher E, Broger L, Mathieu O, Paszkowski J, Vaillant I. Stressinduced activation of heterochromatic transcription. PLoS Genet. 2010;6(10): e1001175.

63. Bosch-Presegue L, Raurell-Vila H, Marazuela-Duque A, Kane-Goldsmith N, Valle A, Oliver J, Serrano L, Vaquero A. Stabilization of Suv39H1 by SirT1 is part of oxidative stress response and ensures genome protection. Mol Cell. 2011;42(2):210-23.

64. Rawlings JS, Rosler KM, Harrison DA. The JAKJSTAT signaling pathway. J Cell Sci. 2004;117(Pt 8):1281-3.

65. Chou TB, Noll E, Perrimon N. Autosomal P[ovoD1] dominant female-sterile insertions in drosophila and their use in generating germ-line chimeras. Development. 1993;119(4):1359-69.

66. Hubbell E, Liu WM, Mei R. Robust estimators for expression analysis. Bioinformatics. 2002;18(12):1585-92.

67. Gautier L, Cope L, Bolstad BM. Irizarry RA: affy-analysis of Affymetrix GeneChip data at the probe level. Bioinformatics. 2004;20(3):307-15.

68. Gentleman RC, Carey VJ, Bates DM, Bolstad B, Dettling M, Dudoit S, Ellis B, Gautier L, Ge Y, Gentry J, et al. Bioconductor: open software development for computational biology and bioinformatics. Genome Biol. 2004;5(10):R80.

\section{Submit your next manuscript to BioMed Central and we will help you at every step:}

- We accept pre-submission inquiries

- Our selector tool helps you to find the most relevant journal

- We provide round the clock customer support

- Convenient online submission

- Thorough peer review

- Inclusion in PubMed and all major indexing services

- Maximum visibility for your research

Submit your manuscript at www.biomedcentral.com/submit
) Biomed Central 